\title{
EXPERIENCES ON STRUCTURAL OPTIMIZATION UNDER UNCERTAINTY IN AEROSPACE COMPONENTS
}

\author{
AITOR BALDOMIR, SANTIAGO HERNÁNDEZ, CARLOS LÓPEZ \& CLARA CID \\ Structural Mechanics Group, School of Civil Engineering, University of A Coruna, Spain
}

\begin{abstract}
Nondeterministic optimization methods have been studied in depth from an academy and industrial point of view. The main drawback when implementing probabilistic optimization methods to complex structures is the computational cost. Many researchers have dedicated their efforts to the development of efficient algorithms to decrease the computational time required by the original formulation of the Reliability Based Design Optimization (RBDO) problems. Among these efficient methodologies the SORA (Sequential Optimization and Reliability Assessment) method has been widely used due to its decoupled formulation. Two application examples are presented with the objective to show the possibilities of taking into account uncertainty data into structural optimization for large-scale engineering problems.
\end{abstract}

Keywords: RBDO, RBTO, aleatory uncertainty, multi-model optimization, reliability index, fail-safe.

\section{INTRODUCTION}

Structural optimization is a common tool used by engineers to improve their designs efficiently [1]-[4]. Generally, the deterministic formulation of the optimization problem is performed using ultimate limit states with their corresponding partial safety coefficients. This approach sometimes leads to oversized structures due to its general treatment of the system uncertainty. A good characterization of the uncertainty affecting structural responses allows us to improve the final design. In that sense Reliability Based Design Optimization (RBDO) methods [5]-[7] have been positioned as the solution to a better link between safety and cost of structures. The main advantages of this approach are the possibility of establishing the target reliability for each specific constraint and consider the real uncertainty that affects to structural parameters independently. On the contrary, the main disadvantage is the computational effort required by these methods when numerous random variables or state limits are involved. RBDO methods were initially applied only to analytical examples and later to more complex numerical problems. Among the structural applications we can find examples in aerospace [8], [9], automobile components [10], [11] or in civil engineering structures like long-span bridges [12], [13].

Although original formulation of RBDO is a nested optimization problem, decoupled methods have proven to be very efficient algorithms to obtain accurate results with a low computational effort, being possible their use in combination with finite element models of industrial applications. In that regards two application examples are presented: first a topology optimization of a simplified aircraft tail fuselage and second a multi-model optimization of a stiffened panel considering partial collapses.

\section{RBDO FORMULATION AND METHODS}

Defining the vector of design variables $\mathbf{d}$ and the set of random variables as $\mathbf{x}$, the general RBDO problem can be formulated as:

$$
\min F\left(\mathbf{d}, \boldsymbol{\mu}_{\mathbf{x}}\right)
$$

subject to 


$$
P\left[G_{i}(\mathbf{d}, \mathbf{x}) \leq 0\right]<P_{f_{i}} \quad i=1, \ldots, m,
$$

where $F$ is the objective function, $G$ is the limit state and $P_{f}$ is the probability of failure. When uncertainty is included it is important to know which values of the random variables are used to evaluate the constraints. As can be seen in Fig. 1 there exist uncertainty in the curves that define the design region. Therefore the probabilistic optimum solution is moved into the design region in order to satisfy the constraints with the probability of failure specified in eqn $(1 \mathrm{~b})$.

There is extensive research about find the optimal solution of problem defined above [6], [7], [14]. This problem can be directly solved by using a two level optimization approach with an inner optimization loop to evaluate the probabilistic constraints and an outer loop for the optimization problem. The most popular two level methods are the Reliability Index Approach (RIA) [5] and the Performance Measurement Approach (PMA) [6]. A flowchart of these methods is presented in Fig. 2.

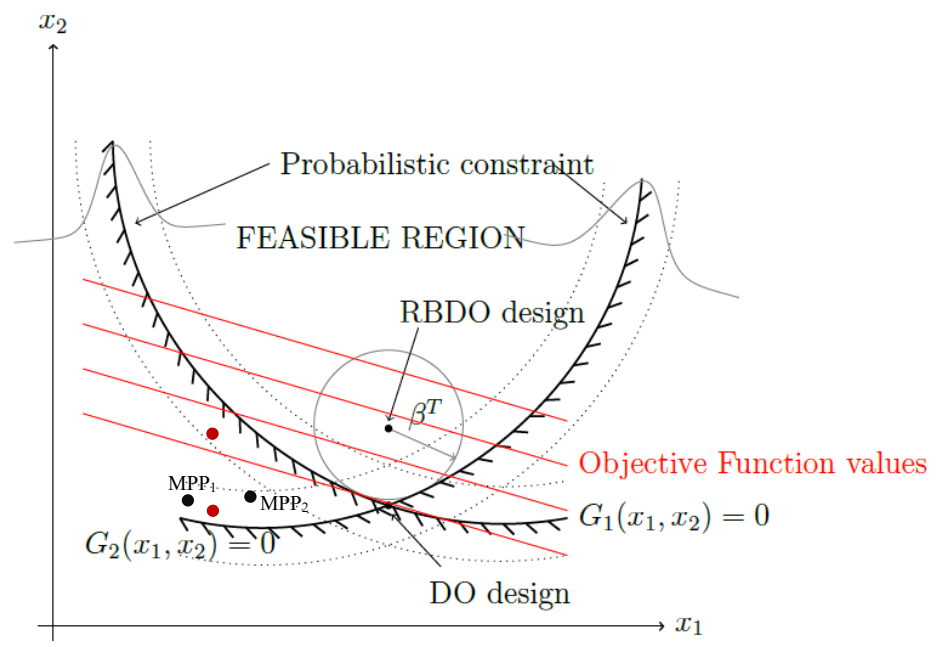

Figure 1: Comparison between RBDO and DO approach.

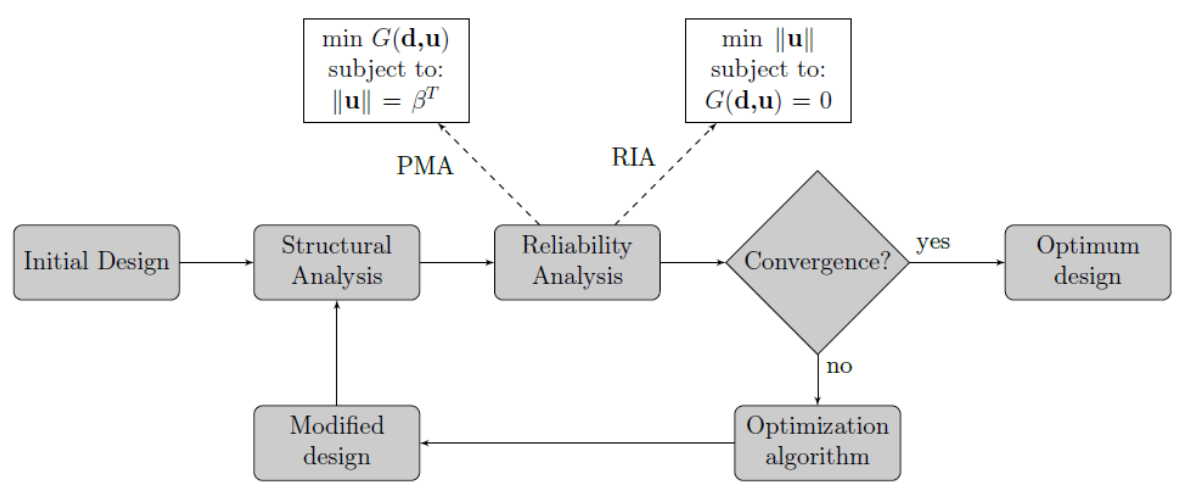

Figure 2: Flowchart of two level methods. 
As indicated in Fig. 2 the reliability analysis consist of a new optimization problem that searchs the Most Probable Point of failure (MPP) that represents the nearest point from the mean value over the limit state surface. This distance measured in the standard normalized $u$-space is defined as the realibility index $\beta[15]$.

As commented initially, these methods require a high computational effort. A very interesting strategy consist of separating the reliability assessment from the DO problem. Thus the RBDO problem becomes in a sequence of deterministic optimizations, each followed by its corresponding reliability analysis. Sequential Optimization and Reliability Assessment (SORA) is one of the most popular decoupled method proposed by Du and Chen [7]. The formulation of this method is the following:

$$
\min F\left(\mathbf{d}, \boldsymbol{\mu}_{\mathbf{x}}\right)
$$

subject to

$$
G_{i}\left(\mathbf{d}, \boldsymbol{\mu}_{\mathbf{x}}-s_{i}, \mathbf{p}_{\mathrm{MPP}}\right) \leq 0
$$

where $\mathbf{x}$ are random design variables and $\mathbf{p}$ are non-design random variables. The first step is to perform the deterministic optimization considering $\mathbf{x}_{\mathbf{M P P}}=\mu_{\mathbf{x}}$ and $\mathbf{p}_{\mathbf{M P P}}=\mu_{\mathbf{P}}$. Vector $\mathbf{s}$ gets to keep the MPP into the deterministic boundary and is defined as $\mathbf{s}=\mu_{\mathbf{x}}-\mathbf{x}_{\mathbf{M P P}}$. Fig. 3 shows a simplified flowchart of this decoupled method.

\section{APPLICATION EXAMPLE 1: TOPOLOGY OPTIMIZATION}

\subsection{Introduction}

Topology optimization is becoming one of the most popular optimization techniques since the emergence of modern fabrication processes as Additive Layer Manufacturing (ALM). These techniques are even applied with metals such as titanium, which allows the fabrication of geometries similar to those obtained by topology optimization. The objective of this work is to include uncertainty at early stages of the design and analyze the effect in subsequent design phases.

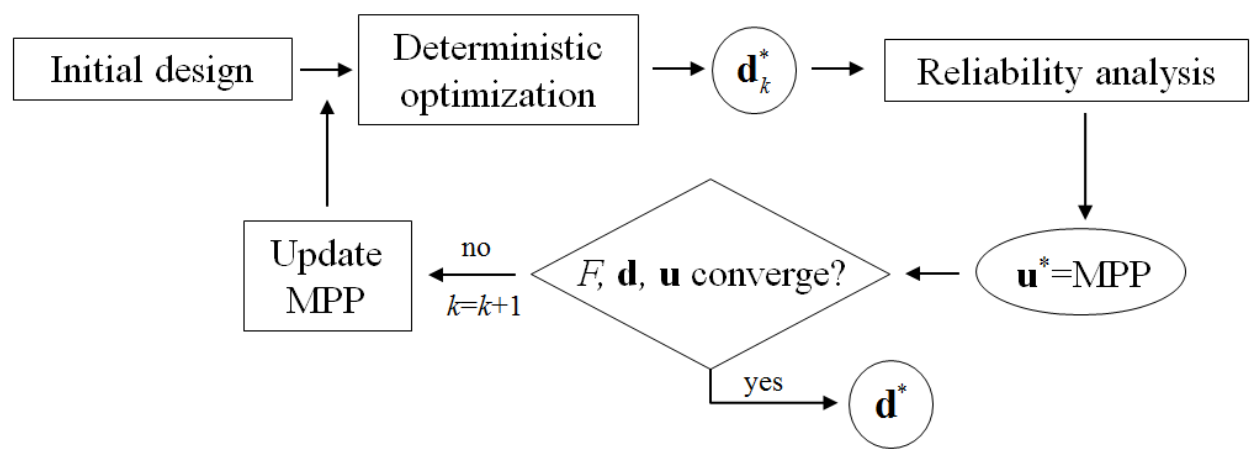

Figure 3: Simplified flowchart of SORA method. 


\subsection{Simplified aircraft tail fuselage}

Fig. 4 shows the FE model with 75978 3D finite elements of a simplified aircraft fuselage previously studied in López et al. [16]. The fuselage is loaded in the rear part of the structure and with the loads transmitted by the vertical and horizontal tail planes (VTP-HTP).

First, the deterministic topology optimization (DTO) is performed with the objective of minimizing the material volume subject to Von Mises stress constraint of $\sigma_{\max }=300 \mathrm{MPa}$. Once the DTO is solved, the weighted compliance is obtained, being considered as constraint in the following RBDO problem.

In this example uncertainty in loads and material are defined as shown in Table 1.

Afterwards, the RBDO problem is defined, being the target to obtain a design as stiff as the preliminary structural scheme provided by the DTO when uncertainty in the load values and Young's modulus are taken into account. The RBDO problem is formulated as:

$$
\begin{gathered}
\min V(\mathbf{d}) \text { s.t. } \\
P\left[W C(\mathbf{d})>W C^{D T O}\right] \leq P_{f}=1.35 \cdot 10^{-3}\left(\beta^{T}=3\right), \\
\sigma(\mathbf{d}) \leq \sigma_{\max },
\end{gathered}
$$

where $\mathrm{WC}^{\mathrm{DTO}}=4.56 \mathrm{E} 7 \mathrm{~mm} / \mathrm{N}$, value obtained in the previous DTO.

Numerical results are presented in Table 2 and the final structural layout is presented in Fig. 5. As can be observed the optimal structural layout is different in both approaches. Thus, considering uncertainty in early stages of the design can lead to a different structure.

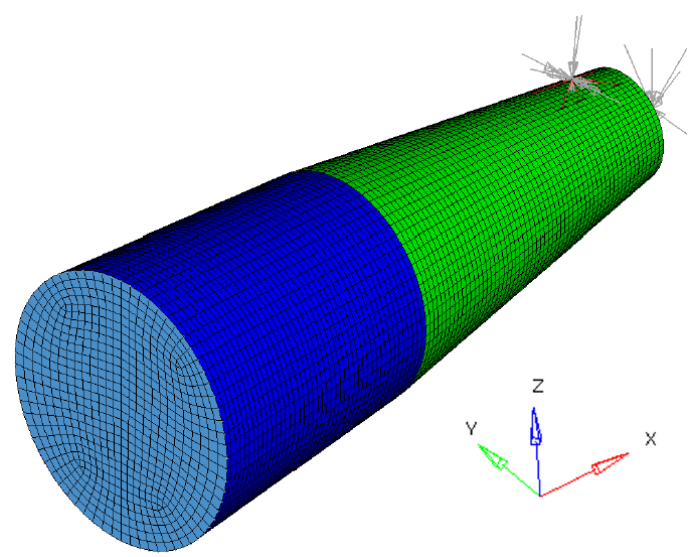

Figure 4: 3D design region of the simplified aircraft fuselage.

Table 1: Definition of the uncertainty data.

\begin{tabular}{lcccc}
\hline Random variable & Distribution & $\mu$ & $\sigma$ & $\delta$ \\
\hline Young's modulus $E(\mathrm{MPa})$ & Log-Normal & 74000 & 3700 & 0.05 \\
Load multiplier factor $\phi_{i}(i=1, \ldots, 6)$ & Normal & 1.0 & 0.1 & 0.1 \\
\hline
\end{tabular}


Table 2: Numerical results.

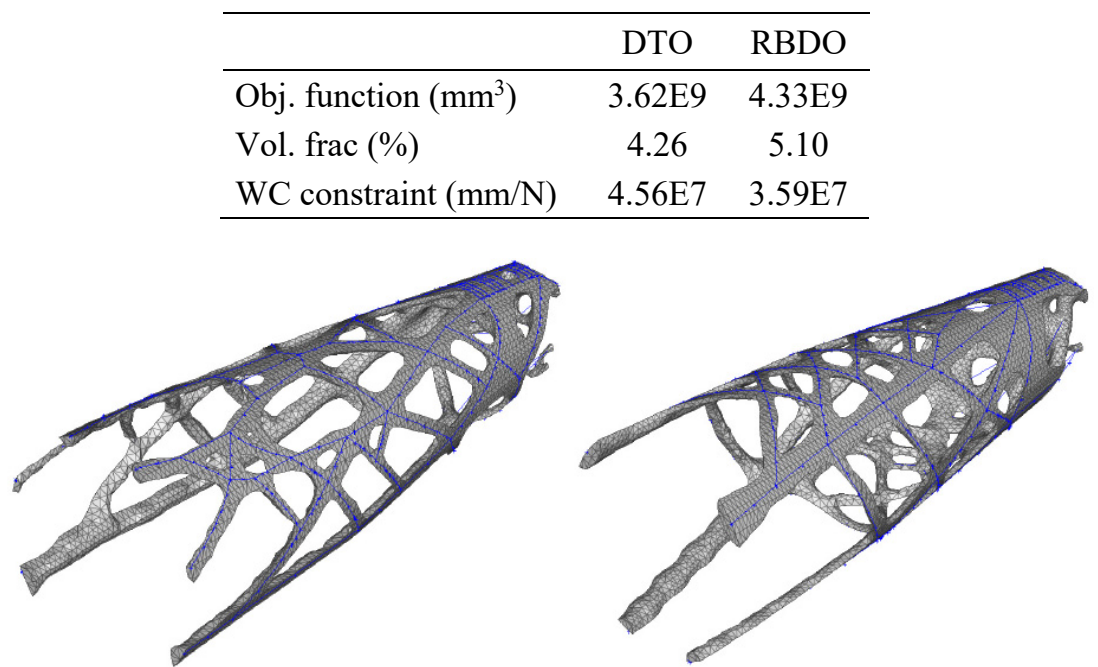

Figure 5: Topology results for DTO (left) and RBTO (right).

The following step is to analyse which structure is better in a subsequent size RBDO with the same target reliability $\left(\beta^{\mathrm{T}}\right)$. The results of both optimization problems can be interpreted in two different internal bar structures as can be seen in Fig. 6 . For both structures the same size RBDO problem is performed as:

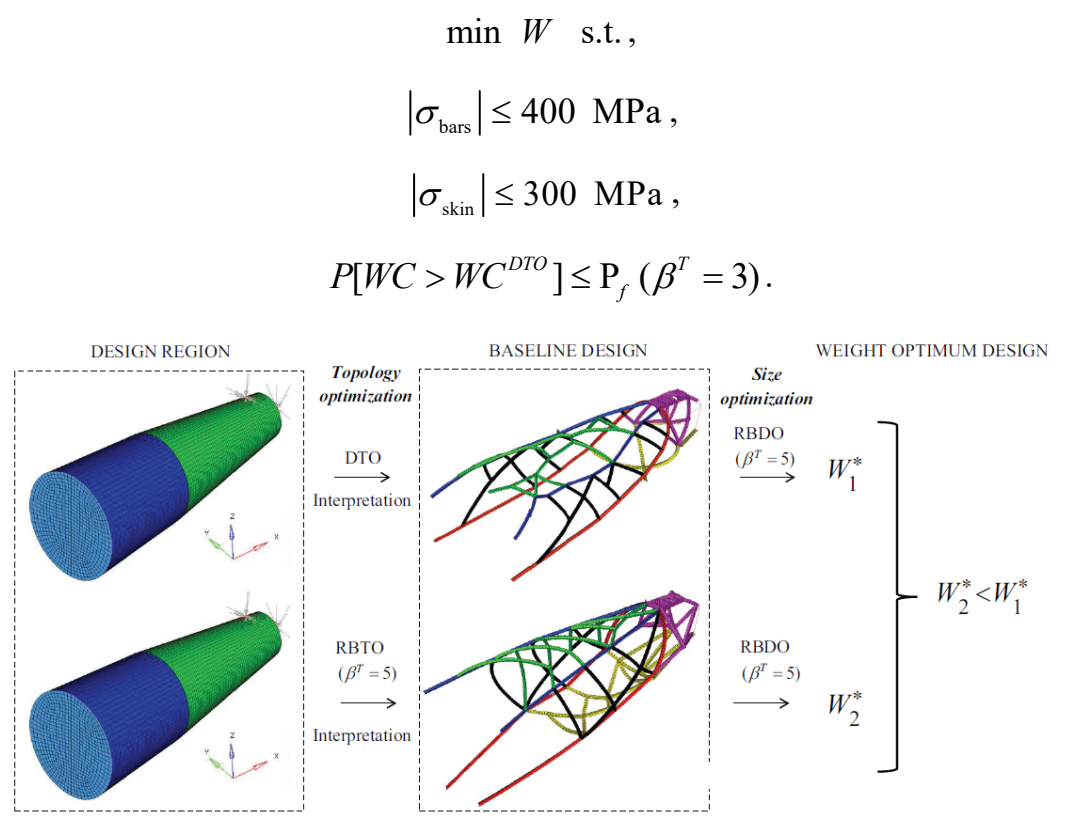

Figure 6: Comparison of designs provided by the RBTO and DTO results. 
Fig. 6 shows the sequence of the entire process of comparison between RBTO and DTO approaches. The idea is to compare the final weight of the same RBDO problem (size optimization) of two different structures. The optimum weight obtained for the model based on DTO and RBDO results were $8.16 \mathrm{kN}$ and $7.35 \mathrm{kN}$ respectively. There is a significant weight reduction in the final design when uncertainty is included in early stages of the design as the topology optimization procedure.

\section{APPLICATION EXAMPLE 2: MULTI-MODEL OPTIMIZATION}

\subsection{Introduction}

Several aerospace regulations contemplate the possibility of accidental damages in aircrafts that produce loss of structural parts in flight. Therefore, the possibility of a partial collapse must be contemplated into the design process of these structures. For that purpose, a set of additional configurations need to be considered into the design process of certain structures apart from the intact model. This strategy is known as multi-model optimization.

\subsection{Curved stiffened panel of an aircraft fuselage}

The Federal Aviation Administration published the Advisory Circular 25.905-1 [17]. This design regulation aims to minimize the hazards that could occur to an airplane if a propeller blade fails and its impact causes a loss of structure in the fuselage. The curved stiffened panel presented [18] is composed by four frames (curved stiffeners), four stringers (longitudinal stiffeners) and the skin. Fig. 7 shows the geometry and the notation used for the definition of stringers and frames profiles, as well as the skin thickness.

Shear and compression loads presented in Fig. 7 are used to perform the buckling analysis of the structure through a FE model defined in Abaqus [19]. The material used was aluminum type AL2024-T3 for the skin and aluminum type AL7075-T6 for the stiffeners. The design variables (d) considered are the set of cross-sectional dimensions of frames, stringers and skin shown in Fig. 8.

Five damaged models $(d=1, \ldots, 5)$ were arbitrarily generated as a result of the removal of part of the panel structure. The proposed incomplete configurations can be seen in Fig. 9, with the corresponding identification number $d$ and the first buckling factor of the initial design.
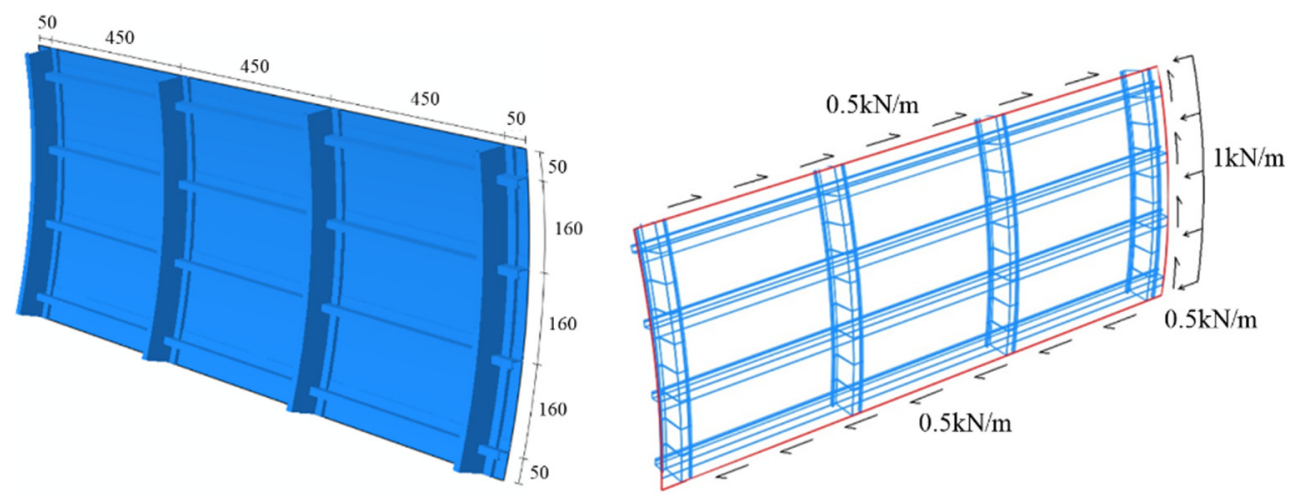

Figure 7: Geometry and loads. Units in $\mathrm{kN}$ and $\mathrm{m}$. 


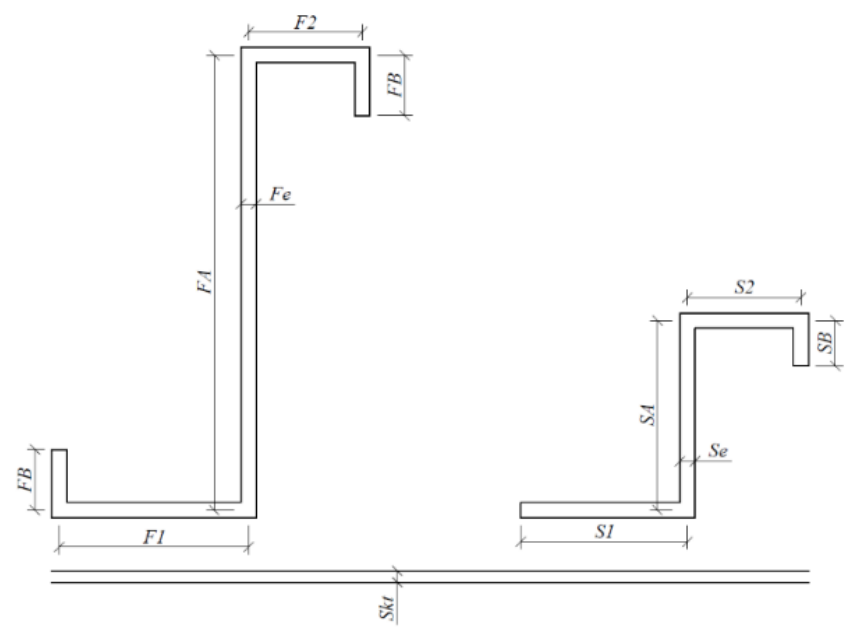

Figure 8: Transversal cross section of frames (left), stringers (right) and skin thickness (bottom).

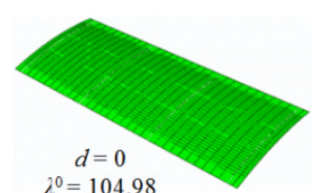

$$
\lambda^{0}=104.98
$$

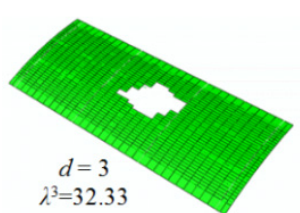

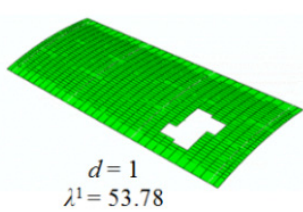

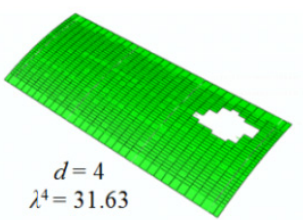

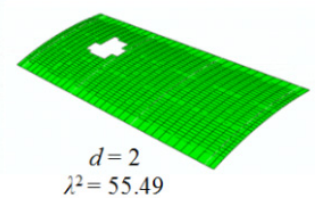

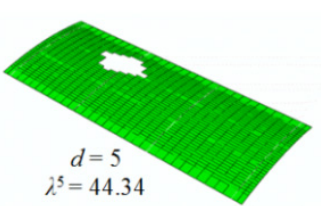

Figure 9: FEM of the panel $(d=0)$ and partial collapses $(d=1, \ldots, 5)$.

Table 3: Mean and standard deviation of the normal random variables.

\begin{tabular}{ccccccc}
\hline & $P_{c}^{0}(\mathrm{kN} / \mathrm{m})$ & $P_{c}^{d}(\mathrm{kN} / \mathrm{m})$ & $P_{s}^{0}(\mathrm{kN} / \mathrm{m})$ & $P_{s}^{d}(\mathrm{kN} / \mathrm{m})$ & $E_{\text {slin }}(\mathrm{MPa})$ & $E_{\text {stifeners }}(\mathrm{MPa})$ \\
\hline$\mu_{x}$ & 1 & 0.6 & 0.5 & 0.3 & 72000 & 71000 \\
$\sigma_{x}$ & 0.1 & 0.06 & 0.05 & 0.03 & 7200 & 7100 \\
\hline
\end{tabular}

The random variables $(\mathbf{x})$ considered are the values of the compression $\left(P_{c}{ }^{0}, P_{c}{ }^{d}\right)$ and shear $\left(P_{s}{ }^{0}, P_{s}{ }^{d}\right)$ loads defined in the buckling analysis, apart from the elasticity modulus of the skin $\left(E_{\text {skin }}\right)$ and the stiffeners $\left(E_{\text {stiffeners }}\right)$ material (Table 3$)$.

The objective is to get the minimum structural mass of the intact structure satisfying probabilistic buckling constraints in both intact and damaged configurations. Thus, the multimodel RBDO problem can be formulated as: 


$$
F=(M F+M S+M S k) /(1.45 \cdot 0.58)\left[\mathrm{kg} / \mathrm{m}^{2}\right]
$$

subject to

$$
\left.\begin{array}{c}
P\left[\lambda^{0}\left(\mathbf{d}, P_{C}^{0}, P_{S}^{0}, E_{\text {skin }}, E_{\text {stiffeners }}\right) / \lambda_{\min }^{0}-1 \leq 0\right] \leq P_{f_{0}}, \\
P\left[\lambda^{d}\left(\mathbf{d}, P_{C}^{d}, P_{S}^{d}, E_{\text {skin }}, E_{\text {stiffeners }}\right) / \lambda_{\text {min }}{ }^{d}-1 \leq 0\right] \leq P_{f_{d}} \quad d=1, \ldots, D, \\
P\left[1-\left|w_{i}^{0}\left(\mathbf{d}, P_{C}^{0}, P_{S}^{0}, E_{\text {skin }}, E_{\text {stiffeners }}\right)\right| / w_{\max } \leq 0\right] \leq P_{f_{0}^{i}} \quad i=1, \ldots, n_{S}, \\
P\left[1-\left|w_{i}^{d}\left(\mathbf{d}, P_{C}{ }^{d}, P_{S}^{d}, E_{\text {skin }}, E_{\text {stiffeners }}\right)\right| / w_{\text {max }} \leq 0\right] \leq P_{f_{d}^{i}} \quad i=1, \ldots, n_{S} \quad d=1, \ldots D, \\
11.423 \leq \frac{F A}{F e} \leq 15.714 ; \quad 11.423 \leq \frac{F 2}{F e} \leq 15.714 ; \quad 3.214 \leq \frac{F B}{F e} \leq 4.286 \\
11.423 \leq \frac{S A}{S e} \leq 15.714 ; \quad 11.423 \leq \frac{S 2}{S e} \leq 15.714 ; \quad 3.214 \leq \frac{S B}{S e} \leq 4.286
\end{array}\right\},
$$

being $M F, M S$ and $M S k$ the mass in kg of the frames, stringers and skin, respectively. The probabilistic constraints are established on the buckling factor $\lambda^{d}$ of each configuration of the panel (eqns (5b) and (5c)) as well as the buckling mode amplitude $w_{i}^{d}$ of the stiffeners (eqns (5d) and (5e)). Slenderness constraints of the cross section in stiffeners (eqn (5f)) are also included as deterministic constraints. The minimum values of buckling factors imposed in eqns (5b) and (5c) are $\lambda^{0}{ }_{\min }=125$ and $\lambda^{d}{ }_{\min }=75$ respectively, and the maximum displacement allowed in the stiffeners (eqns (5d) and (5e)) must be $w_{\max }=0.05$, that is, less than $5 \%$ of the maximum displacement of the buckling mode. The target safety index is set $\beta^{T}=3.719$, corresponding to a probability of failure of $P_{f}=0.0001$. The probabilistic optimization of eqn (5) has initially been solved for the intact model exclusively, $d=0$. Then, more damaged models were added progressively to the existent ones, increasing the size of the probabilistic optimization problem. Results are presented in Table 4.

Table 4: Results of the RBDO considering only the intact model $(d=0)$ and the multimodel configurations $D=2, D=3, D=4$ and $D=5$.

\begin{tabular}{cccccc}
\hline $\begin{array}{c}\mathrm{DV} \\
(\mathrm{mm})\end{array}$ & $d=0$ & $\begin{array}{c}D=2 \\
(d=0,1,2)\end{array}$ & $\begin{array}{c}D=3 \\
(d=0,1,2,3)\end{array}$ & $\begin{array}{c}D=4 \\
(d=0,1,2,3,4)\end{array}$ & $\begin{array}{c}D=5 \\
(d=0,1,2,3,4,5)\end{array}$ \\
\hline$S k t$ & 1.9871 & 2.0806 & 2.4385 & 2.4999 & 2.5023 \\
$F 1$ & 5 & 5 & 5 & 5 & 7.972 \\
$F 2$ & 14.5386 & 14.5386 & 20.2624 & 14.5386 & 25.3471 \\
$F A$ & 20 & 20 & 20.2624 & 20 & 34.8687 \\
$F B$ & 4.0906 & 4.0906 & 4.1443 & 4.0906 & 7.1317 \\
$F e$ & 1.2728 & 1.2728 & 1.2895 & 1.2728 & 2.219 \\
$S 1$ & 5 & 5 & 5 & 5 & 7.0317 \\
$S 2$ & 19.8293 & 20.3639 & 22.8302 & 23.4465 & 23.5314 \\
$S A$ & 27.2781 & 28.0135 & 31.4063 & 32.2541 & 32.3709 \\
$S B$ & 5.5792 & 7.2098 & 6.4236 & 7.1994 & 6.6209 \\
$S e$ & 1.7359 & 1.7827 & 1.9986 & 2.0526 & 2.06 \\
\hline$F\left(\mathrm{~kg} / \mathrm{m}^{2}\right)$ & 7.6865 & 8.0877 & 9.5459 & 9.7951 & 10.711 \\
\hline
\end{tabular}




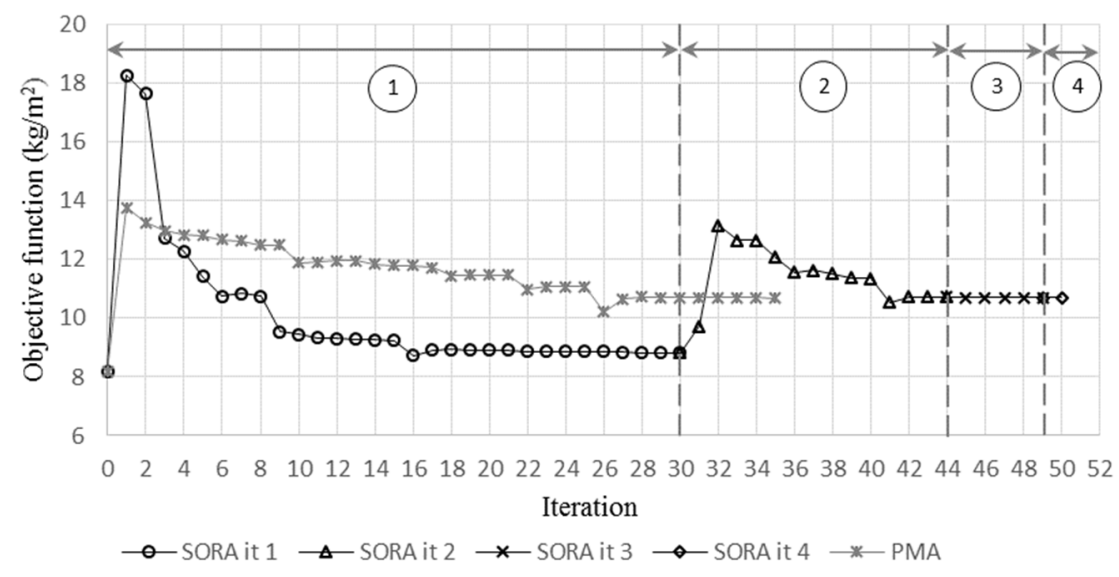

Figure 10: Evolution of the objective function for $D=5$ in SORA and PMA method.

As can be seen in Table 4, the penalty mass of the panel, compared with the optimum design of the intact structure $(d=0)$, is not linear with the number of damages considered, being the determining factor the size and location of the damage. This phenomenon can be appreciated in the volume increment when including the partial collapse 3 and 5. Fig. 10 summarizes the evolution of the objective function after each cycle of the SORA method in the optimization problem with $D=5$, as well as the evolution of the objective function using PMA method. From Table 4 it can be drawn that although the optimum mass of the panel increases progressively by adding damaged configurations, the same does not happen with the value of the design variables. For instance, comparing the optimum design for $D=2$ and $D=3$, all the values of the design variables increase except the vertical border height of the stringers $(S B)$. Moreover, the values of the design variables $F 2, F A, F B$ and $F e$ when $D=4$ decrease considerably in comparison with the corresponding values when $D=3$.

\section{CONCLUSIONS}

Probabilistic optimization problems can be efficiently solved with complex structural analyses or even with different optimization strategies. In this work two different application examples have been presented in order to visualize the effect of considering uncertainty data in topology optimization or in fail-safe design processes.

\section{ACKNOWLEDGMENT}

The research leading to these results is part of the research project DPI2016-76934-R financed by the Spanish Ministry of Economy and Competitiveness.

\section{REFERENCES}

[1] Vanderplaats, G., Numerical Optimization Techniques for Engineering Design, 3rd ed., VR \& D Inc., 2001.

[2] Arora, J., Introduction to Optimum Design, 3rd ed., Academic Press, 2012.

[3] Hernández, S. \& Fontán, A.N., Practical Applications of Design Optimization, WIT Press: Southampton and Boston, 2002.

[4] Haftka, R. \& Gurdal, Z., Elements of Structural Optimization, 3rd ed., Kluwer Academic Publishers, 1991. 
[5] Enevoldsen, I. \& Sørensen, J.D., Reliability-based optimization in structural engineering. Struct. Saf., 15(3), pp. 169-196, 1994.

[6] Tu, J., Choi, K.K. \& Park, Y.H., A new study on reliability-based design optimization. J. Mech. Des., 121(4), pp. 557-564, 1999.

[7] Du, X. \& Chen, W., Sequential optimization and reliability assessment method for efficient probabilistic design. J. Mech. Des., 126(2), pp. 225-233, 2004.

[8] López, C., Bacarreza, O., Baldomir, A. \& Hernández, S., Reliability-based design optimization of composite stiffened panels in post-buckling regime. Struct. Multidiscip. Optim., 55(3), pp. 1121-1141, 2016.

[9] D’Ippolito, R., Ito, K., van der Heggen, B. \& Tzannetakis, N., Probabilistic optimization of the transmission loss of a composite ribbed panel. 4th International Conference on Uncertainty in Structural Dynamics, Leuven, Belgium, Code 107115, 2012.

[10] Moustapha, M., Sudret, B., Bourinet, J.-M. \& Guillaume, B., Quantile-based optimization under uncertainties using adaptive Kriging surrogate models. Struct. Multidiscip. Optim., 54(6), pp. 1403-1421, 2016.

[11] Cid Montoya, M., Costas, M., Díaz, J., Romera, L.E. \& Hernández, S., A multiobjective reliability-based optimization of the crashworthiness of a metallic-GFRP impact absorber using hybrid approximations. Struct. Multidiscip. Optim., 52(4), pp. 827-843, 2015.

[12] Kusano, I., Baldomir, A., Jurado, J.A. \& Hernández, S., Reliability based design optimization of long-span bridges considering flutter. J. Wind Eng. Ind. Aerodyn., 135, pp. 149-162, 2014.

[13] Saad, L., Aissani, A., Chateauneuf, A. \& Raphael, W., Reliability-based optimization of direct and indirect LCC of RC bridge elements under coupled fatigue-corrosion deterioration processes. Eng. Fail. Anal., 59, pp. 570-587, 2016.

[14] Aoues, Y. \& Chanteauneuf, A., Benchmark study of numerical methods for reliabilitybased design optimization. Struct. Multidiscip. Optim., 41(2), pp. 277-294, 2010.

[15] Cornell, C.A., A probability based structural code. J. Am. Concr. Inst., 66(12), pp. 974-985, 1969.

[16] López, C., Baldomir, A. \& Hernández, S., Deterministic versus reliability-based topology optimization of aeronautical structures. Struct. Multidiscip. Optim., 53(4), pp. 907-921, 2016.

[17] U.S. Department of Transportation, Minimizing the Hazards from Propeller Blade and Hub Failures, Federal Aviation Administration, Advisory Circular, AC 25.905-1, 2000.

[18] Cid Bengoa, C., Baldomir, A., Hernández, S. \& Romera, L., Multi-model reliabilitybased design optimization of structures considering the intact configuration and several partial collapses. Struct. Multidiscip. Optim., 57(3), pp. 977-994, 2018.

[19] Abaqus, Abaqus 6.14.2. Documentation, 2014. 